\title{
Layer Number Contrast of CVD-derived Graphene in Low Voltage Scanning Electron Microscopy
}

Jining Xie and James Spallas

Agilent Technologies, 4330 W. Chandler Boulevard, Chandler, AZ 85226, USA.

Characterization of graphene films by scanning electron microscopy (SEM) is a rapid, non-invasive and effective imaging technique which is complimentary to other techniques such as optical microscopy, atomic force microscopy, Raman spectroscopy, etc. However, SEM imaging of graphene is difficult mainly due to the intrinsic features of graphene films including their ultra thin layers, smooth surface and fine morphology. Imaging graphene films with a low beam voltage field emission SEM is attractive due to its unique combination of high resolution, a small beam/specimen interaction volume, enhanced contrasts and the capability of revealing more surface details. The object of this work is to study the thickness contrast observed in low voltage SEM imaging of CVD-derived graphene thin films. Imaging in this work was performed at $1000 \mathrm{~V}$ acceleration voltage with a microchannel plate (MCP) detector.

The properties of graphene films vary with the number of layers [1]. Particularly for microelectronic applications, identification of the number of graphene layers is needed. The as grown graphene films synthesized by CVD usually contain multi-layer domains [2]. Figure 1a is a typical SEM micrograph displaying some features of the graphene film and the $\mathrm{Cu}$ foil beneath. Comparing with high voltage SEM imaging $(>5 \mathrm{kV})$, low voltage imaging exhibits the multi-layer domains with enhanced contrasts [3]. As shown in Figure 1b, four areas with decreasing brightness can be attributed to monolayer, bilayer, trilayer, and quadrilayer graphene films, respectively [4]. The SE intensity profile along the yellow line is shown in Figure 1c. Obviously, four levels can be identified based on their signal intensities. This result implies that the signal intensity coming from the graphene film depend upon the number of layers. Comparison between an SE image of a multi-layer domain and its corresponding topo image indicates that this contrast can not be solely elucidated as the topographic contrast (Figure 1d).

In order to build up the relationship between signal intensities and graphene thicknesses (Figure 2a), a CASINO Monte Carlo simulation [5] was conducted for a $1 \mathrm{kV}$ beam with $10 \mathrm{~nm}$ spot size on quadrilayer graphene $(\sim 1.4 \mathrm{~nm}$ thick $) / \mathrm{Cu}$ substrate. Figure $2 \mathrm{~b}$ depicts the energy distribution by position which confirms the penetration of incident electrons through the graphene and the interaction with the substrate. It can be seen that, under the current condition in this study, a significant part of the beam/specimen interaction volume is located inside the $\mathrm{Cu}$ substrate. As a consequence, the secondary electrons emitted from the $\mathrm{Cu}$ substrate actually contribute for the detected signal. The thickness contrast is hypothesized to be primarily induced by the attenuation of secondary electrons emitted from the $\mathrm{Cu}$ substrate by the graphene layers. As illustrated in Figure 3a, a higher number of graphene layers result in a lower signal intensity. This hypothesis is based on several assumptions: 1) the interaction volume difference in $\mathrm{Cu}$ beneath graphene with a few layers is negligible; 2) there is no inelastic attenuation in the outermost graphene layer; 3) no diffraction effect from the crystalline graphene needs to be considered. For the electron attenuation mechanism, the detected signal intensity should decreases exponentially with the increasing number of graphene layers which can be described as: $I_{N}=A e^{-N \cdot \frac{d_{0}}{\lambda}}+B$, where $I_{N}, d_{0}$ and $\lambda$ are the SE intensity detected after attenuation by $\mathrm{N}$ layers of graphene, the thickness of graphene monolayer and the electron inelastic mean free path, respectively. A 
and B are fitting parameters. By using the intensity values extracted from Figure 1c, we plot the curve of intensity vs. number of layers, as shown in Figure 3b. For such graphene layers observed in this sample, the curve matches well with an exponential formula, indicative of the correctness of the hypothesis.

In summary, low voltage SEM imaging can not only resolve fine features of CVD-graphene films, but also differentiate graphene layers with different thicknesses based on the observed contrasts [6].

References:

[1] E McCann et al, Physical Reviews B 74, (2006), p. 161403(R).

[2] I Vlassiouk et al, ACS Nano 5 (2011), p. 6069.

[3] H Hiura et al, Applied Physics Express 3 (2010), p. 095101.

[4] X Li et al, Science 324 (2009), p.1312.

[5] D Drouin et al, Scanning 29 (2007), p. 92.

[6] The authors acknowledge Dr. Ying Feng and Professor Ke Chen from Temple University for helpful discussions and contributions to this work.
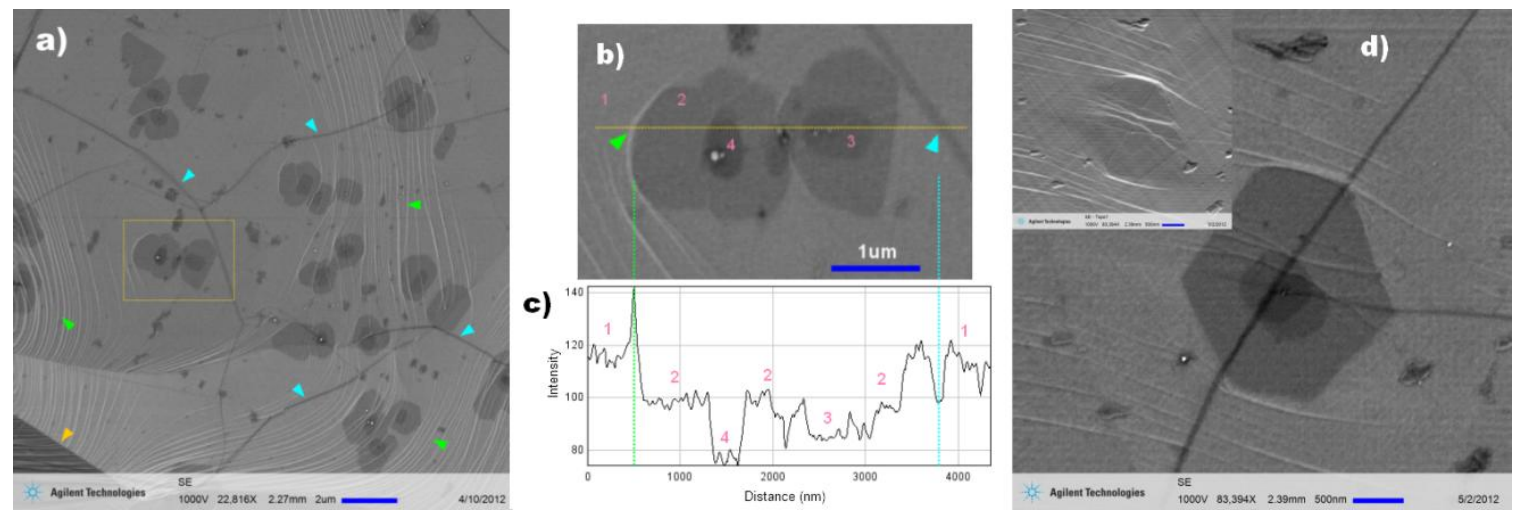

Figure 1. (a-b) Typical images of a CVD synthesized graphene on $\mathrm{Cu}$; (c) the intensity profile along the yellow line; (d) comparison of an SE image and corresponding topo image (inset).

a)

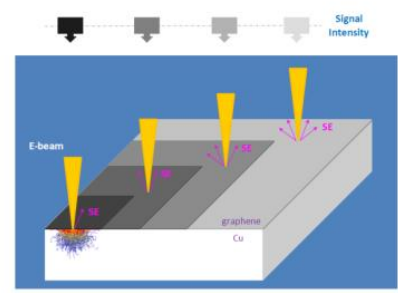

b)

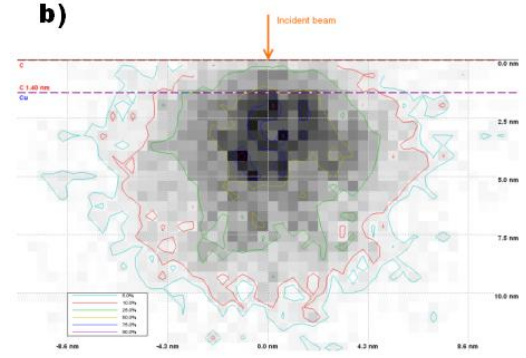

Figure 2. a) Schematic of graphene thickness contrast; b) Monte Carlo simulation result.

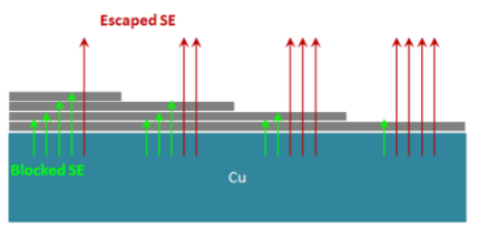

b)

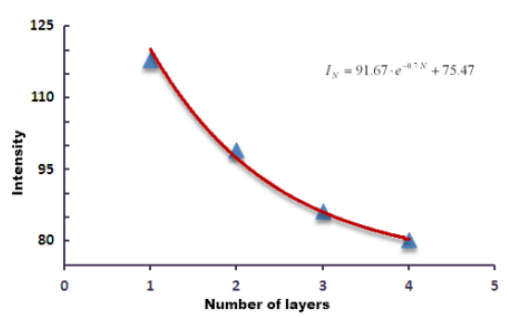

Figure 3. (a) schematic of electron attenuation; b) plot of intensity vs. number of graphene layers. 